\title{
Characteristics and Diversity of Cyanobacteria in Periphyton from Lentic Tropical Ecosystem, Brazil
}

\author{
Sirlene Aparecida Felisberto ${ }^{1}$, Danúzia Batista da Silva e Souza² \\ ${ }^{1}$ Botanical Department, Federal University of Goiás (UFG), Goiânia, Brazil \\ ${ }^{2}$ Specialization and Postgraduate Institute (IEPG), Faculty Oswaldo Cruz, Goiânia, Brazil \\ Email: fsirfe@gmail.com
}

Received 30 September 2014; revised 30 October 2014; accepted 14 November 2014

Copyright (C) 2014 by authors and Scientific Research Publishing Inc.

This work is licensed under the Creative Commons Attribution International License (CC BY). http://creativecommons.org/licenses/by/4.0/

(c) (i) Open Access

\begin{abstract}
This study aimed to perform the taxonomic survey of the species of periphytic Cyanobacteria in a lentic tropical environment, seeking to contribute to the knowledge on biodiversity and their distribution. This study was conducted at the Samambaia Reservoir, which is located at Federal University of Goiás, Goiânia, Central West region of Brazil. In general, the water of the Samambaia Reservoir is characterized as more turbid and the increased biochemical oxygen demand as well as a higher concentration of coliforms in the rainy season. Twenty six samples were collected, ten in the dry season $(09 / 2010$ and $11 / 2010 ; 07 / 2012)$ and ten in the rainy season $(01 / 2011$ and 03/2011; 01/2013). Periphyton was collected from stems of aquatic plants (Cyperaceae), which were in the marginal area of the reservoir. With the floristic survey, 38 taxa of Cyanobacteria were identified in periphyton of the Samambaia Reservoir. Phormidium (family Phormidiaceae) and Aphanocapsa (Merismopediaceae) are among genera with the highest species richness. Regarding morphological types, many species of filamentous cyanobacteria were identified, followed by colonial taxa. In relation to the frequency of occurrence and the seasonal period, 14 taxa were classified in the rare category, 12 in common category and 12 in constant category. Regarding the seasonal period, Cyanophyceae were the greatest wealth in the rainy season (97.4\%).
\end{abstract}

\section{Keywords}

Filamentous Cyanophyceae, Reservoir, Goiás State, Taxonomy

\section{Introduction}

Brazil is the country that has the largest amount of freshwater in the world, and the greatest river system in Goiás is one of the most important water divisors and contributors in the "composition" of water resources of 
the country, with tributaries and palm swamps which join to form the three major Brazilian watersheds [1].

Despite the significance of the amount of freshwater to Goiás Cerrado, there is little research on periphytic communities [2]-[8].

In these periphytic communities, there are important photosynthetic microorganisms, such as Cyanobacteria (blue algae), which are found in the community of periphytic algae or planktonic. These microorganisms become more populous because they are capable of forming massive growth of blooms with adverse effects on sanitation, which are aggravated when toxic cyanobacterial species are present [9]. However, the knowledge of their morphological variability (important for their identification) is still little known, which requires a thorough revision [10].

Traditionally, based on morphology Cyanobacteria have been classified into four orders (Chroococcales, Oscillatoriales, Nostocales and Stigonematales) [11] [12].

The basic morphology comprises unicellular, colonial and multicellular filamentous forms.

Unicellular forms, such as Chroococcales, have spherical, ovoid or cylindrical cells, and in some species the colony forms are constituted by a cell aggregate held together by the slimy matrix secreted during the growth of the colony [13].

Filamentous morphology is the result of repeated cell divisions occurring in a single plane at right angles to the main axis of the filament and consisting of a chain of cells is called a trichome, which may be straight or coiled [14].

Filamentous species in the order Oscillatoriales, with unseriated and unbranched trichomes, are composed of essentially identical cells. Other orders with a filamentous organization (Nostocales and Stigonematales) are characterized with trichomes having a heterogeneous cellular composition, which may be differentiated into heterocysts (vegetative cell somewhat larger and rounder shape, diminished pigmentation, thicker cell envelopes, and usually prominent cyanophycin granules at poles adjacent to other cells) and akinetes (vegetative cell larger, thicker cell envelopes, containing many reserve granules) [14] [15].

Therefore, this study aimed to execute the taxonomic survey of the unicellular, colonial and multicellular filamentous species of periphytic Cyanobacteria in a lentic tropical environment.

\section{Materials and Methods}

The study area $\left(16^{\circ} 35^{\prime} \mathrm{S}, 49^{\circ} 16^{\prime} \mathrm{W}\right.$, Figure 1) is located in Campus II of the Federal University of Goiás in Goiânia-GO, Central West region of Brazil.

The Samambaia Reservoir was constructed in 1972 by damming the stream Samambaia [16]; it has an average depth of $3.5 \mathrm{~m}$ and was characterized as an environment oligo to mesotrophic. This reservoir has been built to the purpose of fish farming experiments of Federal University of Goiás (UFG) and later went on to supply water for the entire university campus through the company of Goiás Sanitation S/A (Saneago). However, there are significant points of sources and diffusion of pollutants.

The characterization of drought and rain was based on climatological daily data of rainfall obtained through newsletters from the Rainfall Station of Goiânia, located in the School of Agronomy and Food Engineering, UFG, distant about $600 \mathrm{~m}$ of the study area.

In general, the water of the Samambaia Reservoir is more turbid with the increased of biochemical oxygen demand, as well as a higher concentration of coliforms in the rainy season (Table 1, [8]). The climate in the study area is considered as tropical by according to Köppen classification [17].

Periphyton was collected from stems of aquatic plants (Cyperaceae) that were in the marginal area of the reservoir (Three petioles per sample point), five sampling points in the study area. Twenty samples were collected, ten in the dry season $(24 / 09 / 2010$ and $04 / 11 / 2010 ; 07 / 2012)$ and ten in the rainy season $(06 / 01 / 2011$ and 02/03/2011;01/2013).

The periphytic substrate material was removed with the aid of a razor blade, followed by distilled water jets. After the scraping, the material was transferred to vials, preserved and fixed with Transeau solution.

The samples were deposited in the herbarium collection of the Federal University of Goiás, Goiânia-GO under the numbering 47324 - 47349.

The samples were analyzed between slide and cover slip in the preparation of about 10 temporary slides per sample. The capture images of subjects was processed with the aid of the optical microscope ZEISS brand (model AXIOSKOP 40) and a digital camera coupled by the optical system of the microscope.

Identification of taxa was made by the morphological and morphometric characteristics of the cells, which 


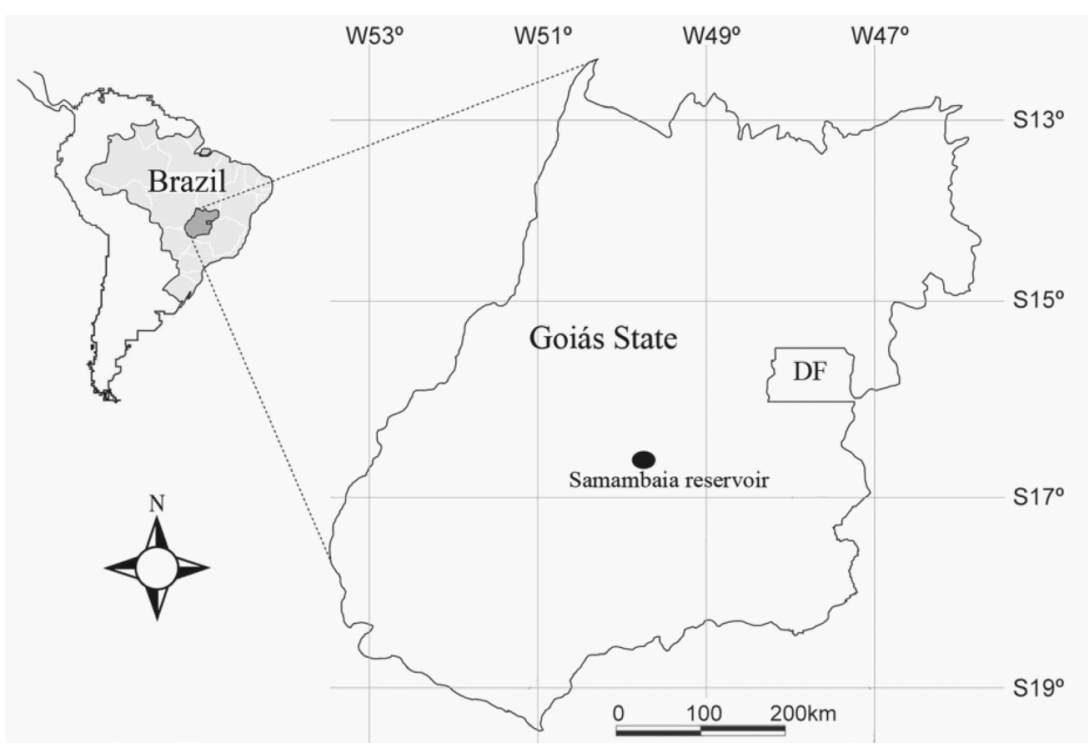

Figure 1. Map showing the location of the research area Samambaia Reservoir in Goiás State.

Table 1. Values of abiotic variables of dry and wet periods at the Samambaia Reservoir, Goiânia-GO. Source: [8].

\begin{tabular}{ccc}
\hline Variables & Dry Period & Rain Period \\
\hline $\mathrm{pH}$ & $7.6 \pm 0.2$ & $6.9 \pm 0.2$ \\
Water Temperature $\left({ }^{\circ} \mathrm{C}\right)$ & $24 \pm 3.6$ & $25 \pm 0$ \\
Turbidity $(\mathrm{NTU})$ & $7.9 \pm 1.6$ & $32.2 \pm 0.3$ \\
Dissolved Oxygen $(\mathrm{mg} / \mathrm{L})$ & $7.3 \pm 0.5$ & $6 \pm 0.4$ \\
Biochemical Oxygen Demand $(\mathrm{mg} / \mathrm{L})$ & $1.9 \pm 2$ & $3 \pm 0.4$ \\
Index of Total Coliforms $(\mathrm{CFU} / \mathrm{g})$ & $4479 \pm 4206$ & $19,598 \pm 6502$ \\
Index of Fecal Coliforms $(\mathrm{CFU} / \mathrm{g})$ & $642.3 \pm 655.6$ & $2143 \pm 1319$ \\
Nitrate $(\mathrm{mg} / \mathrm{L})$ & $90 \pm 70$ & $40 \pm 40$ \\
Ammonia Nitrogen $(\mathrm{mg} / \mathrm{L})$ & $40 \pm 50$ & $10 \pm 0$ \\
Electrical Conductivity $(\mathrm{mS} / \mathrm{cm})$ & $56 \pm 10.5$ & $62.8 \pm 10.8$ \\
Total Phosphorus $(\mathrm{mg} / \mathrm{L})$ & $20 \pm 20$ & $20 \pm 10$ \\
\hline
\end{tabular}

were obtained by the optical microscope at the increase of 40 to 100 times, and consultation based into the specialized literature, including articles, reviews and monographs. The frequency of occurrence, in percent, was calculated considering the presence and absence of the taxon and divided by the total number of taxa sampled. The categories used were: Rare $(\leq 10 \%)$, Common $(>10 \% \leq 30 \%)$ and Constant $(>30 \%)$.

\section{Results and Discussion}

With the taxonomic analysis, we identified 38 taxa of Cyanobacteria in periphyton of the Samambaia Reservoir (Table 2). Phormidium and Aphanocapsa were the most specious genera (Table 2).

For periods, the higher taxonomic richness (97.4\%) was evaluated for the rainy season. Regarding morphological types, many species of filamentous cyanobacteria were identified, followed by colonial taxa.

In relation the frequency of occurrence and the seasonal period, 14 taxa were classified in the rare category,

12 in common category and 12 in constant category (Table 2).

Family Chamaesiphonaceae

Geitleribactron periphyticum Komárek, Plant Syst Evol. 123: 263-281. 1975. 
Table 2. Frequency of occurrence of the species found in the sampling in both study periods of the Samambaia Reservoir. * $=$ absence of species; $\mathrm{x}=$ presence of species; $\mathrm{R}=$ rare $\mathrm{Cm}=$ common; $\mathrm{Cs}=$ constant; Freq. $=$ Frequency in $\%$.

\begin{tabular}{|c|c|c|c|}
\hline Taxa & Dry Period & Rain Period & Freq. \\
\hline Aphanocapsa annulata & $\mathrm{x}$ & $\mathrm{x}$ & $4 \mathrm{Cm}$ \\
\hline Aphanocapsa delicatissima & * & $\mathrm{x}$ & $2 \mathrm{R}$ \\
\hline Aphanocapsa elachista & $\mathrm{x}$ & $\mathrm{x}$ & $9 \mathrm{Cs}$ \\
\hline Aphanocapsa koordersii & $\mathrm{x}$ & $\mathrm{x}$ & $15 \mathrm{Cs}$ \\
\hline Aphanothece conglomerata & $\mathrm{x}$ & $\mathrm{x}$ & $7 \mathrm{Cm}$ \\
\hline Arthrospira jenneri & $\mathrm{x}$ & $*$ & $1 \mathrm{R}$ \\
\hline Chroococcus dispersus & $\mathrm{x}$ & $\mathrm{x}$ & $11 \mathrm{Cs}$ \\
\hline Chroococcus minutus & $\mathrm{x}$ & $\mathrm{x}$ & $8 \mathrm{Cs}$ \\
\hline Coelomoron tropicale & $\mathrm{x}$ & $\mathrm{x}$ & $14 \mathrm{Cs}$ \\
\hline Coelosphaerium evidenter-marginatum & $*$ & $\mathrm{x}$ & $2 \mathrm{R}$ \\
\hline Coelosphaerium kuetzingianum & $\mathrm{x}$ & $\mathrm{x}$ & $5 \mathrm{Cm}$ \\
\hline Dolichospermum sp. & $\mathrm{x}$ & $\mathrm{x}$ & $4 \mathrm{Cm}$ \\
\hline Epigloeosphaera brasilica & $*$ & $\mathrm{x}$ & $1 \mathrm{R}$ \\
\hline Fischerella sp. & $*$ & $\mathrm{x}$ & $1 \mathrm{R}$ \\
\hline Geitleribactron periphyticum & $\mathrm{x}$ & $\mathrm{x}$ & $5 \mathrm{Cm}$ \\
\hline Geitlerinema amphibium & $\mathrm{x}$ & $\mathrm{x}$ & $5 \mathrm{Cm}$ \\
\hline Geitlerinema splendidum & $\mathrm{x}$ & $\mathrm{x}$ & $7 \mathrm{Cm}$ \\
\hline Geitlerinema unigranulatum & $\mathrm{x}$ & $\mathrm{x}$ & $9 \mathrm{Cs}$ \\
\hline Komvophoron schmidlei & $\mathrm{x}$ & $\mathrm{x}$ & $2 \mathrm{R}$ \\
\hline Limnothrix guttulata & $*$ & $\mathrm{x}$ & $4 \mathrm{Cm}$ \\
\hline Microcystis natans & $\mathrm{x}$ & $\mathrm{x}$ & $2 \mathrm{R}$ \\
\hline Nostoc sp. & $\mathrm{x}$ & $\mathrm{x}$ & $7 \mathrm{Cm}$ \\
\hline Oscillatoria limosa & $\mathrm{x}$ & $\mathrm{x}$ & $2 \mathrm{R}$ \\
\hline Oscillatoria princeps & $\mathrm{x}$ & $\mathrm{x}$ & $10 \mathrm{Cs}$ \\
\hline Phormidium autumnale & $\mathrm{x}$ & $\mathrm{x}$ & $6 \mathrm{Cm}$ \\
\hline Phormidium chlorinum & * & $\mathrm{x}$ & $1 \mathrm{R}$ \\
\hline Phormidium irriguum & $\mathrm{x}$ & $\mathrm{x}$ & $14 \mathrm{Cs}$ \\
\hline Phormidium lucidum & $\mathrm{x}$ & $\mathrm{x}$ & $5 \mathrm{Cm}$ \\
\hline
\end{tabular}

Figure 2(2) and Figure 2(3)

Cells heteropolar, solitary or in groups, elongated, attached by one end to substrate (usually by indistinct, hyaline, gelatinous pad), and in groups or in stellate clusters. Cells $(5.2-7.4 \times 2.2 \mu \mathrm{m})$ are oval to cylindrical and rod-shaped, rounded at the apex, slightly and shortly attenuated or rounded at the base.

Family Chroococcaceae

Chroococcus dispersus (Keissl.) Lemmerm, Ark. Bot. 2: 102. 1904.

Figure 2(4) and Figure 2(5)

Colonies microscopic, usually 4 - 16 cells, gathered in free groups in the center; mucilage hyaline, forming an indistinct wide margin around the cells; cells spherical (2.6 - $4.2 \mu \mathrm{m}$ in diameter); without aerotopes.

Chroococcus minutus (Kützing) Nägeli, Gatt. Einz. Algen: 46. 1849.

Figures 2(6)-(9)

Colonies microscopic, usually 2 - 4 cells, with mucilage around; cells semispherical (3.6 - $6.2 \mu \mathrm{m}$ in diameter) without aerotopes.

Family Merismopediaceae

Aphanocapsa annulata G. McGregor, Nova Hedwigia 84: 299. 2007. 

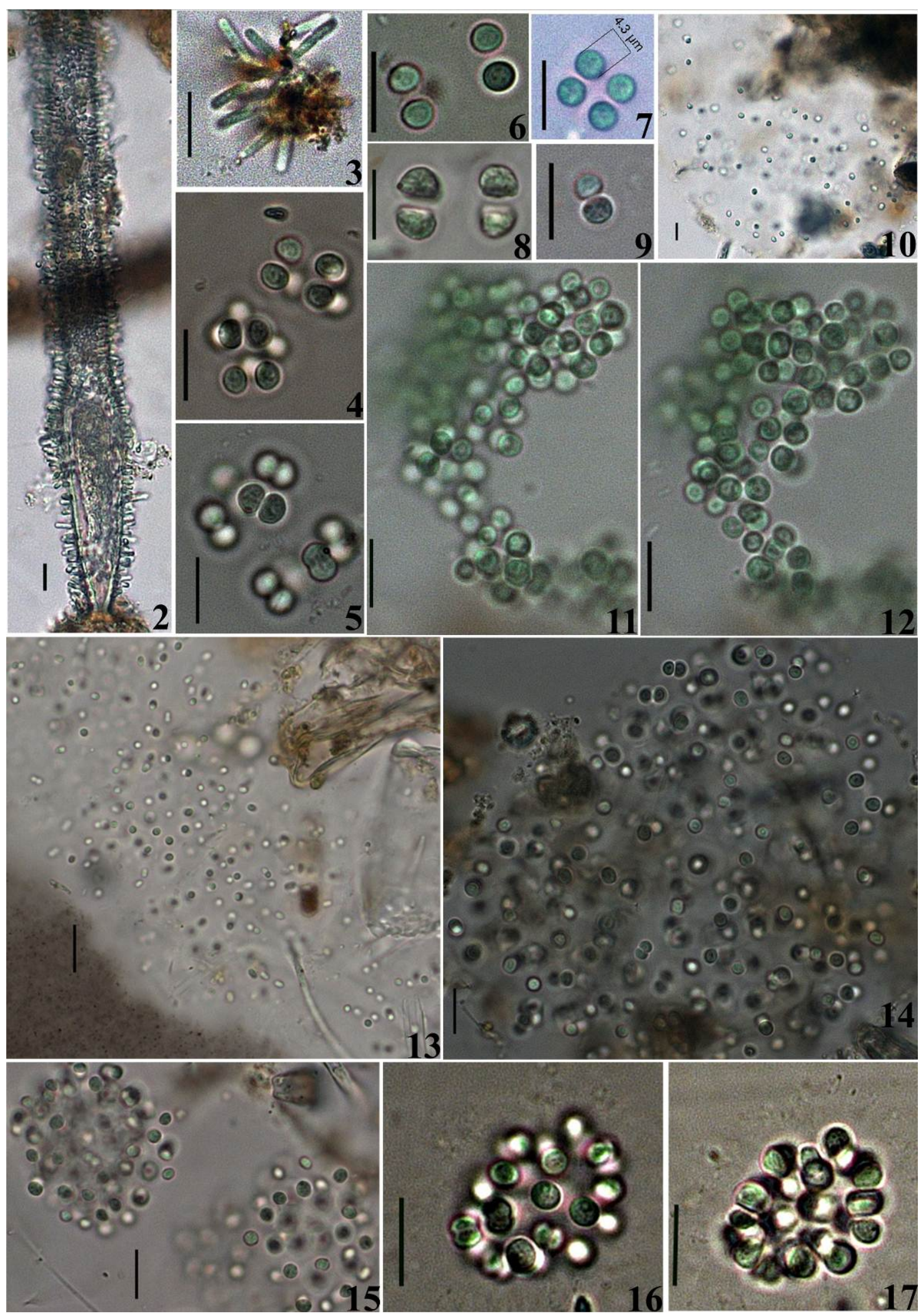

Figure 2. 2-3. Geitleribactron periphyticum; 4-5. Chroococcus dispersus; 6-9. C. minutus; 10. Aphanocapsa delicatissima; 11-12. A. annulata; 13. A. elachista; 14-15. A. koordersii; 16-17. Coelomoron tropicale. Scale bar $=10$ $\mu \mathrm{m}$. 
Figure 2(11) and Figure 2(12)

Colonies irregularly elongated; cells spherical (2.2 - $4.0 \mu \mathrm{m}$ in diameter) densely arranged; mucilage hyaline. Aphanocapsa delicatissima W. West \& G.S. West, J. Linn. Soc. Bot. 40: 431. 1912.

Figure 2(10)

Colonies spherical; cells spherical (1.6 - $2.9 \mu \mathrm{m}$ in diameter), loosely and irregularly arranged; mucilage hyaline.

Aphanocapsa elachista W. West \& G.S. West, Journ. Linn. Soc. 30: 276.1894.

Figure 2(13)

Colonies spherical to elongated; cells spherical, $(2.4-3.3 \mu \mathrm{m}$ in diameter) loosely and irregularly arranged; mucilage hyaline.

Aphanocapsa koordersii Strøm, Nyt Mag. Naturv. 61:128. 1923.

Figure 2(14) and Figure 2(15)

Colonies subspherical and uneven; cells spherical (2.2 - $3.2 \mu \mathrm{m}$ in diameter), loosely and irregularly arranged; there are subcolonies; cells generally with a central bead; mucilage hyaline.

Coelomoron tropicale Senna, A. C. Peres \& Komárek, Nova Hedwigia 67: 96. 1998.

Figure 2(16) and Figure 2(17)

Colonies spherical; cells oval $(2.1-3.6 \mu \mathrm{m}$ in diameter), radially distributed on the surface of the colony; without aerotopes.

Coelosphaerium evidenter-marginatum Azevedo \& Sant'Anna, Algol. Studies 94: 36. 1999.

Figure 3(18) and Figure 3(19)

Colonies spherical, hollow, thick mucilage; cells spherical (2.2 - 3.4 $\mu \mathrm{m}$ in diameter), evenly distributed on the surface of the colony; without aerotopes.

In the specimens found, we observed a thick mucilage, different from the material analyzed by Azevedo and Sant'Anna [18] and Sant'Ana et al. [19], which are mentioning an inconspicuous mucilage hyaline in this species.

Coelosphaerium kuetzingianum Naegeli, Gatt. Einzell. Alg. 54. 1849.

Figure 3(20) and Figure 3(21)

Colonies spherical, hollow, cells oval (2.2 - $3.7 \mu \mathrm{m}$ in diameter), more crowded at the end of the colony; mucilage clear.

\section{Family Gomphosphaeriaceae}

Woronichinia ruzickae Komárek \& Hindák, Arch. Hydrobiol. Suppl. 80 (Algol. Stud. 50-53): 216.1988.

Figure 3(22) and Figure 3(23)

Colonies irregular (15 $\mu \mathrm{m}$ in diameter); cells oval (2.3 - 2.6 $\mu \mathrm{m}$ in diameter); radially and densely arranged in the peripheral layer, which are organized into rods, parallel radiating from the center of colonies.

\section{Family Synechococcaceae}

Aphanothece conglomerata Rich, Trans. R. Soc. S. Afr. 20:185. 1932.

Figure 4(51)

Colonies rounded to elongated, irregular or with cells distributed in the central part of colony; mucilage hyaline, conspicuous; oblong cells $(3.5-6.5 \times 1.7-4.8 \mu \mathrm{m})$, granulated, with aerotopes.

Radiocystis geminata Skuja, Symb. Bot. Upsal. 9(3): 44. 1948.

Figure 4(52)

Colonies subspherical, slightly elongated; cells radially arranged subcolonies, spherical to slightly elongated (4.1 - $4.8 \times 2.6-4.0 \mu \mathrm{m})$; mucilage hyaline; small granules present; without aerotopes.

Epigloeosphaera brasilica Azevedo et al., Hoehnea, 30(3): 285-295. 2003.

Figure 4(53)

Colonies spherical, composed from 2 few hemispherical subcolonies; envelope homogeneous mucilaginous; cells rod-like $(1.4-2.9 \times 1.1-1.4 \mu \mathrm{m})$, sparcely and irregularly distributed on the colony surface.

Family Microcystaceae

Microcystis natans Lemmermann ex Skuja, Acta Horti. Bot. Univ. Latv. 7: 45. 1934.

Figure 3(24)

Colonies spherical, elongated, irregular, $(63 \times 34 \mu \mathrm{m})$, cells spherical $(2.3 \mu \mathrm{m}$ in diameter $)$, presence of aerotopes; mucilage homogeneous.

Family Borziaceae 

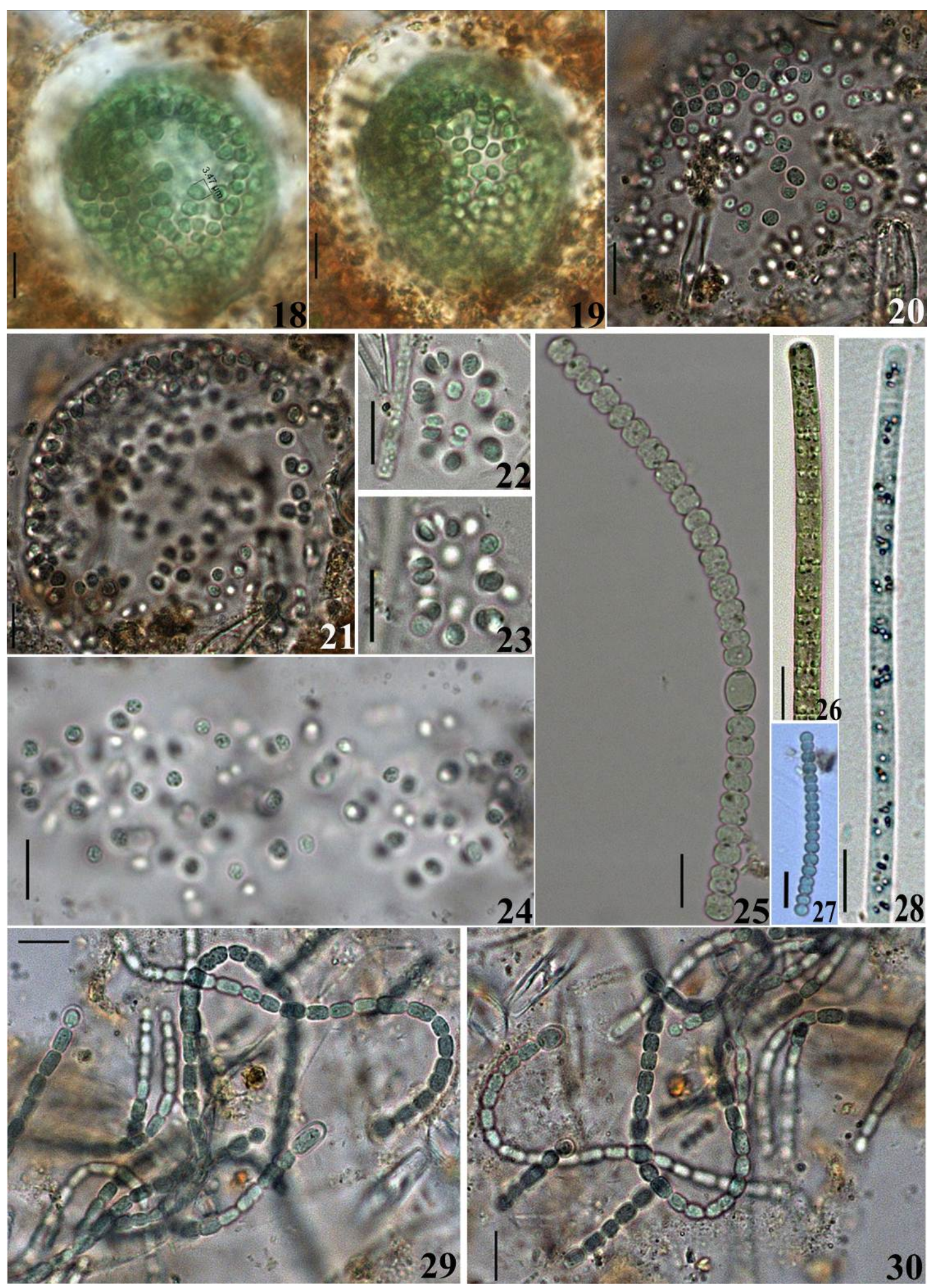

Figure 3. 18-19. Coelosphaerium evidenter-marginatum; 20-21. C. kuetzingianum; 22-23. Woronichinia ruzicka; 24. Microcystis natans; 25. Dolichospermum sp.; 26. Phormidium tergestinum; 27. Komvophoron schmidlei; 28. Limnothrix guttulata; 29-30. Nostoc sp. Scale bar $=10 \mu \mathrm{m}$. 


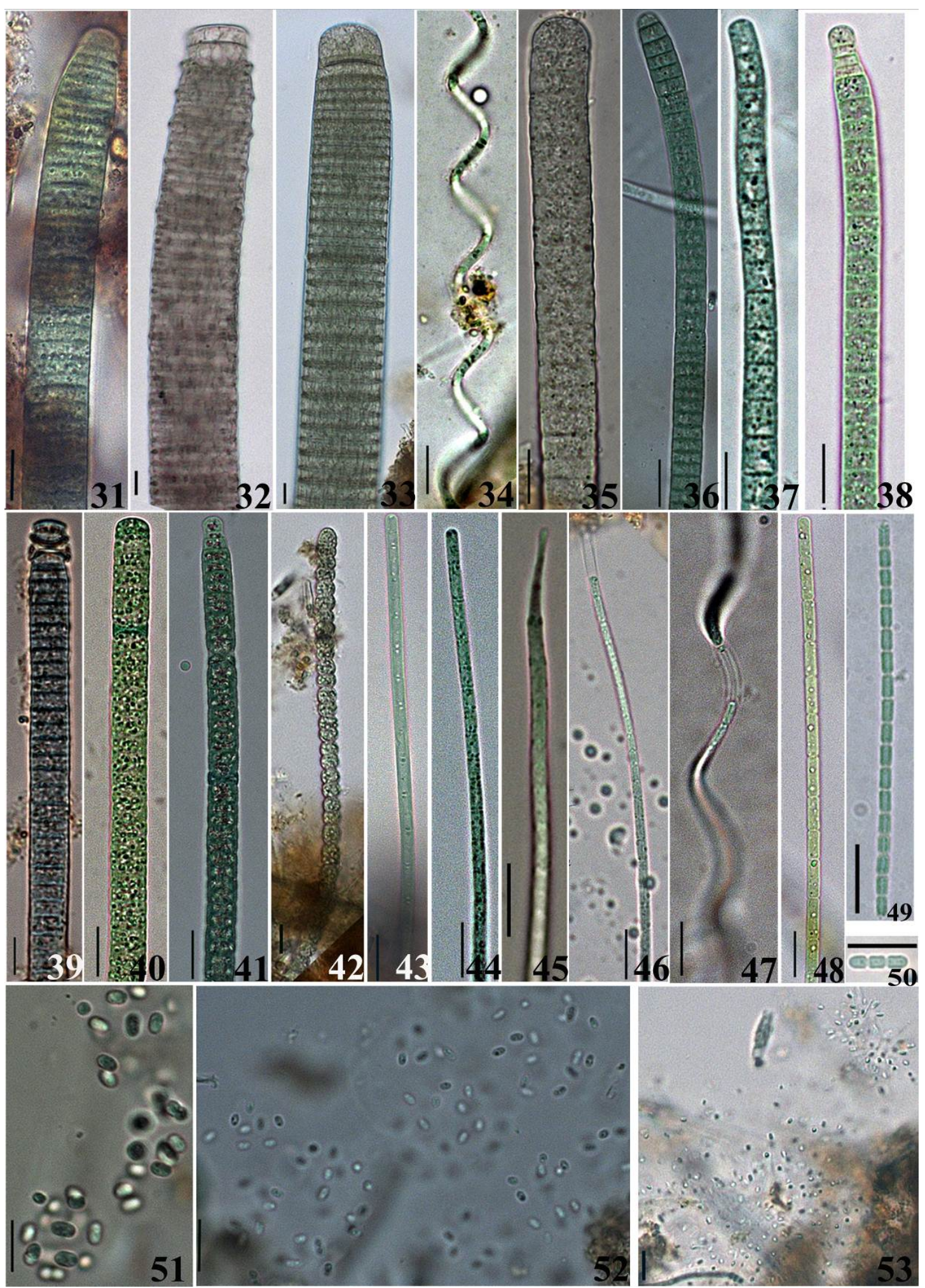

Figure 4. 31. Oscillatoria limosa; 32-33. O. princeps; 34. Arthrospira jenneri; 35. Phormidium cf. lucidum; 36, 38. P. autumnale; 37. P. chlorinum; 39. P. irriguum; 40. P. tergestinum; 41. Planktothix rubescens; 42. Trichodesmium lacustre; 43. Geitlerinema unigranulatum; 44. G. amphibium; 45. G. splendidum; 46. Planktolyngbya limnetica; 47. P. regularis; 48-49. Pseudanabaena catenata; 50. P. mucicola; 51. Aphanothece conglomerata; 52. Epigloeosphaera brasilica; 53. Radiocystis geminata. Scale bar $=10 \mu \mathrm{m}$. 
Komvophoron schmidlei (Jaag) Anagnostidis \& Komárek, Archiv für Hydrobiologie, Supplement 80: 327472. 1988.

Figure 3(27)

Filaments solitary, short, constricted, not attenuated, septum not granulated; cells barrel-shaped $(3.3-4 \times 4.6$ $\mu \mathrm{m})$, without aerotopes; apical cell with conical-obtuse-rounded apex.

Family Oscillatoriaceae

Oscillatoria limosa Agardh ex Gomont, Annales des Sciences Naturelles, Botanique, Série 7, 16: 210. 1892.

Figure 4(31)

Filaments moving, straight, long, not constricted septa; apex straight or slightly curved; cells shorter than long (3.6 - $4.1 \times 11.1-14.7 \mu \mathrm{m})$, granular contents, without aerotopes. cylindrical, apical cell rounded, without calyptra.

Oscillatoria princeps Vaucher ex Gomont, Annales des Sciences Naturelles, Botanique, Série 7, 16: 206. 1892.

Figure 4(32) and Figure 4(33)

Filaments straight, slightly attenuated at the apex, not capitated, without restraint, without granulations in septa, without aerotopes; apical cell hemispherical, presence or absence of calyptra; cells shorter than long (2.8 $5.2 \times 28.5-45.9 \mu \mathrm{m})$.

Family Phormidiaceae

Arthrospira jenneri Stizenberger ex Gomont, Annales des Sciences Naturelles, Botanique, Série 7, 16: 91264. 1892.

Figure 4(34)

Filaments solitary, regularly coiled, $22.2 \mu \mathrm{m}$ in distance between the coils, not constricted at the septum (not shown). Pale bluish-green color. Cells possibly longer than wide (1.7 $\mu \mathrm{m}$ in width), homogeneous structure with irregularly disposed granules, without aerotopes. Apical cells rounded. Sheaths absent or facultatively present, fine and colorless.

Phormidium autumnale (Agardh) Trevisan ex Gomont, Annales des Sciences Naturelles, Botanique, Série 7, 16: 187.1982.

Figure 4(36) and Figure 4(38)

Filaments solitary, straight or flexuous, curved; attenuated to the apex, constricted; cells wider than long (2.8 $3.9 \times 5.7-7.2 \mu \mathrm{m}$ ), filled with granules; apical cell rounded, sometimes truncate, sometimes with calyptra.

Phormidium chlorinum (Kützing ex Gomont) Umezaki \& M. Watanabe, Jap. J. Phycol. 42: 194. 1994.

Figure 4(37)

Filaments solitary, straight or flexuous, curved; attenuated to the apex, constricted; cells wider than long (3.0 $5.4 \times 5.4-6.5 \mu \mathrm{m})$, filled with granules; apical cell rounded.

Phormidium irrigum (Kützing ex Gomont) K. Anagnostidis \& J. Komárek, Arch. Hydrobiol. Suppl. Algol. Stud. 50-53: 405. 1988.

Figure 4(39)

Filaments solitary, straight or flexuous, curved; attenuated to the apex, constricted; cells wider than long (3.0 $4.6 \times 10-11.3 \mu \mathrm{m}$ ), filled with granules in septum; apical cell conical-rounded; without calyptra.

Phormidium lucidum (C. Agardh) Kützing 1843: 194, nom. inval.

Figure 4(35)

Filaments solitary, straight; attenuated to the apex, constricted; cells wider than long $(3.6-4.3 \times 11.5-11.8$ $\mu \mathrm{m})$, filled with granules; apical cell rounded; without calyptra.

Phormidium tergestinum (Kützing) Anagnostidis \& Komárek, Algolog. Stud. 50-53: 406, 1988.

Figure 3(26) and Figure 4(40)

Filaments solitary, straight or flexuous, not attenuated, trichomes constrict or not; cells cylindrical (3.0 - $6.5 \times$ 4.8 - $7.5 \mu \mathrm{m}$ ); apical cell rounded; granular cell content.

Planktothrix rubescens Anagnostidis \& Komárek, J. Archiv für Hydrobiologie, Supplement 80: 327-472. 1988.

Figure 4(41)

Filaments solitary, more or less straight or slightly waved; trichomes constrict or not, cells cylindrical, wider than long $(2.6-4.6 \times 6.8-11.6 \mu \mathrm{m})$, sometimes slightly tapering to the end; cells with aerotopes; end cells (when fully developed) widely rounded or slightly narrowed and with thickened outer cell wall with calyptra. 
Trichodesmium lacustre Klebahn, Forsch Ber. Biol. Stn Plön 3:13. 1895.

Figure 4(42)

Filaments solitary, straight, not attenuated, constricted; cells wider than long (3.9 - $4.9 \times 5.7-7.3 \mu \mathrm{m})$; apical cell rounded, without calyptra; granular cell content, without aerotopes.

The absence of aerotopes was also documented by Komarek and Anagnostidis [20].

\section{Family Nostocaceae}

\section{Dolichospermum sp.}

Figure 3(25)

Filaments moniliformis, straight or slightly curved; sheath mucilaginous wide; cells spherical $(10.6 \times 12.0$ $\mu \mathrm{m})$, with aerotopes; heterocytes spherical $(9.6 \times 6.7 \mu \mathrm{m})$.

\section{Nostoc sp.}

Figure 3(29) and Figure 3(30)

Filaments medley, trichomes straight or flexuous; mucilage hyaline difluente; cells barrel-shaped to spherical (3.3 - $5.4 \times 3-3.7 \mu \mathrm{m})$; heterocytes spherical $(4.4-10.1 \times 4.4 \mu \mathrm{m})$, distributed at the end of the trichome.

\section{Family Pseudanabaenaceae}

Geitlerinema amphibium (Agardh ex Gomont) Anagnostidis, P1. Syst. \& Evol. 164(1/4): 33. 1989.

Figure 4(44)

Filaments straight, rarely curved, not constricted septum, translucent; cells longer than wide $(4.0-8.4 \times 1.7$ $2.6 \mu \mathrm{m}$ ); provided with one bead in the division of cells (i.e., in the septum); apical cell rounded.

Geitlerinema splendidum (Greville ex Gomont) Anagnostidis, P. Syst. Evol. 164: 35. 1989.

\section{Figure 4(45)}

Filaments straight or slightly coiled, not constricted; ends of the trichomes more or less bent, attenuated, sometimes screw-like or in a sickle; cells longer than wide $(1.6-2.7 \times 4.7-8.1 \mu \mathrm{m})$, containing granules; apical cell striking attenuated, spherically capitated; without aerotopes; intense wavelike motility.

Geitlerinema unigranulatum (Singh) Komárek \& Azevedo, Algol. Stud. 99: 51. 2000

Figure 4(43)

Filaments straight, straight or slightly curved, not constricted, not attenuated; cells longer than wide, (3.1 - 7.0 $\times 1.2-1.5 \mu \mathrm{m}$ ); apical cell cylindrical with rounded end; cross wall with one granule; motility present.

Limnothrix guttulata (Goor) Umezaki \& M. Watanabe, Jap. J. Phycol. 42: 207. 1994.

Figure 3(28)

Filaments flexile, not constricted septum, inconspicuous; apex not attenuate; cells more or less isodiametric or up to two times longer than wide $(5.2-7.2 \times 2.4-4.5 \mu \mathrm{m})$ aerotopes scattered irregularly throughout the cell content; apical cell cylindrical, rounded.

Planktolyngbya limnetica (Lemmermann) Komárková-Legnerová \& Cronberg, Arch. Hydrobiol. Suppl. 95 (Algolog. Stud. 67): 23. 1992.

Figure 4(46)

Filaments straight or slightly curved, not constricted septum; apex not attenuated; thin sheath, colorless, conspicuous; cells cylindrical $(1.9-2.6 \times 1.5-2.0 \mu \mathrm{m})$; without aerotopes, sometimes with solitary granules.

Planktolyngbya regularis Komárková-Legnerová \& Tavera, Arch. Hydrobiol./Algolog. Stud. 83: 403-422; 419. 1996.

Figure 4(47)

Filaments slightly curved, coiled, not constricted; apex not attenuated; thin sheath, colorless, conspicuous; cells cylindrical $(5.2 \times 2.1 \mu \mathrm{m})$; without aerotopes, sometimes with solitary granules.

Pseudanabaena catenata Lauterborn, Verhandlungen des Naturhistorisch-Medizinischen Vereins zu Heidelberg, 2, 13: 437. 1915.

Figure 4(48) and Figure 4(49)

Filaments flexuous, translucent, constricted septum; cylindrical cells (4.5 - $6.0 \times 1.6-2.3 \mu \mathrm{m})$; homogeneous content, clearly distinguishable in chromate and centroplasm; apical cell cylindrical, rounded or slightly truncated conical.

Pseudanabaena mucicola (Nauman \& Hubber-Pestalozzi) Bourrelly, Les Algues d'Eau Douce, III: 440. 1970.

Figure 4(50)

Filaments straight, constricted, low mobility, trembling, with a defined number of cells (up to 6); cells qua- 
dratic and cylindrical $(2.9-3.7 \times 1.6-2.2 \mu \mathrm{m})$, granules sometimes present; apical cell cylindrical, rounded.

\section{Family Scytonemaceae}

\section{Scytonema sp.}

Figures 5(54)-(57)

Filaments with false branching; trichomes of cylindrical cells inside firm sheaths; cells longer than board, as long as wide or wider than long $(4.6-10.7 \times 6.9-19.2 \mu \mathrm{m})$. Filaments are often coiled and form entangled mats. Heterocysts spherical $(15.4 \times 15.9 \mu \mathrm{m})$.

\section{Family Stigonemataceae}

\section{Fischerella sp.}

Figure 5(58) and Figure 5(59)

Filaments with true branches, cells spherical to cylindrical, generally longer than broad (6.1 - $15.4 \times 6.1-9.2$ $\mu \mathrm{m})$, particularly those distal from the base, which may taper. Heterocysts spherical $(8.5 \times 6.9 \mu \mathrm{m})$ or even compressed (shorter than broad) and intercalary.
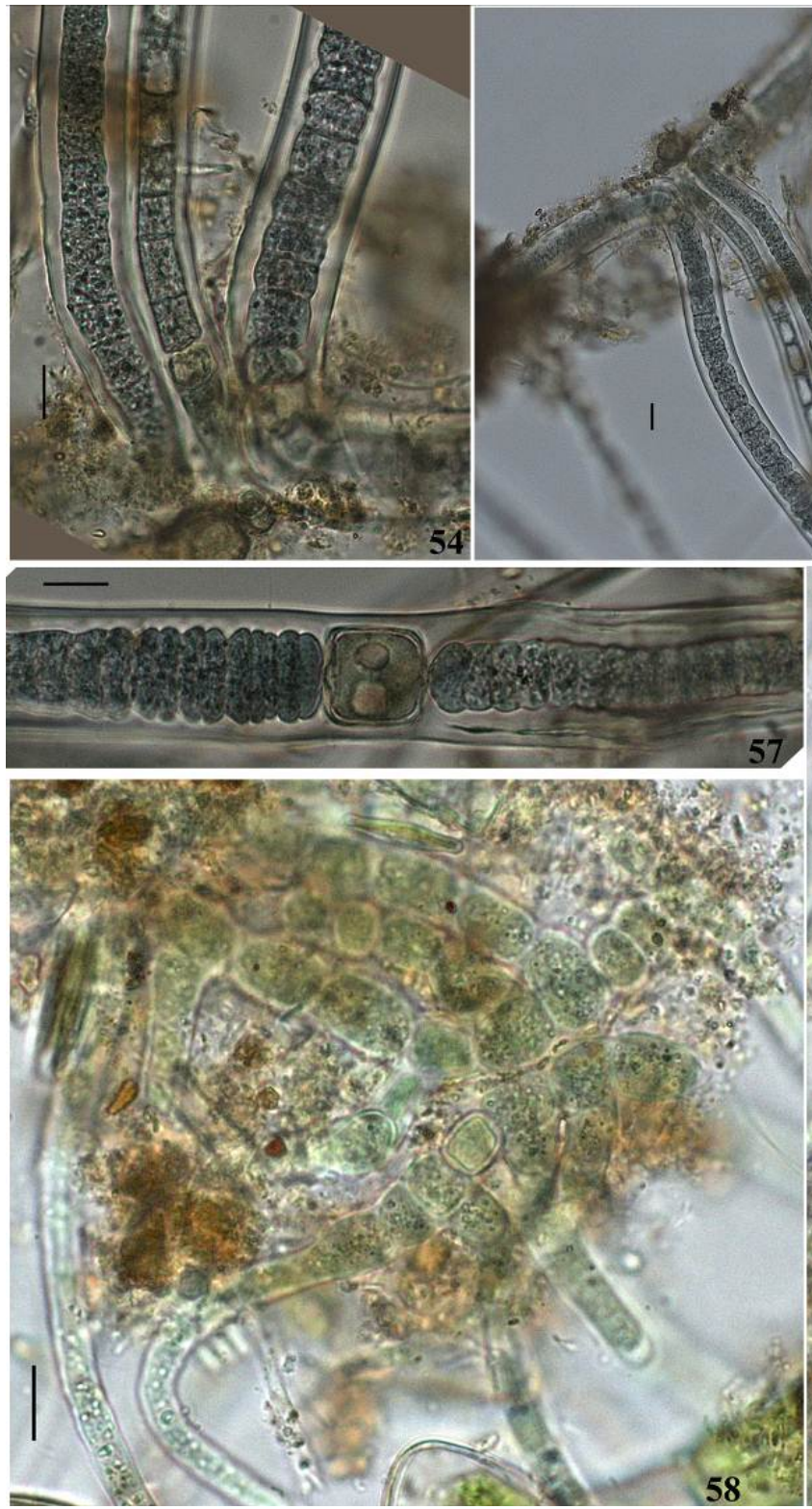
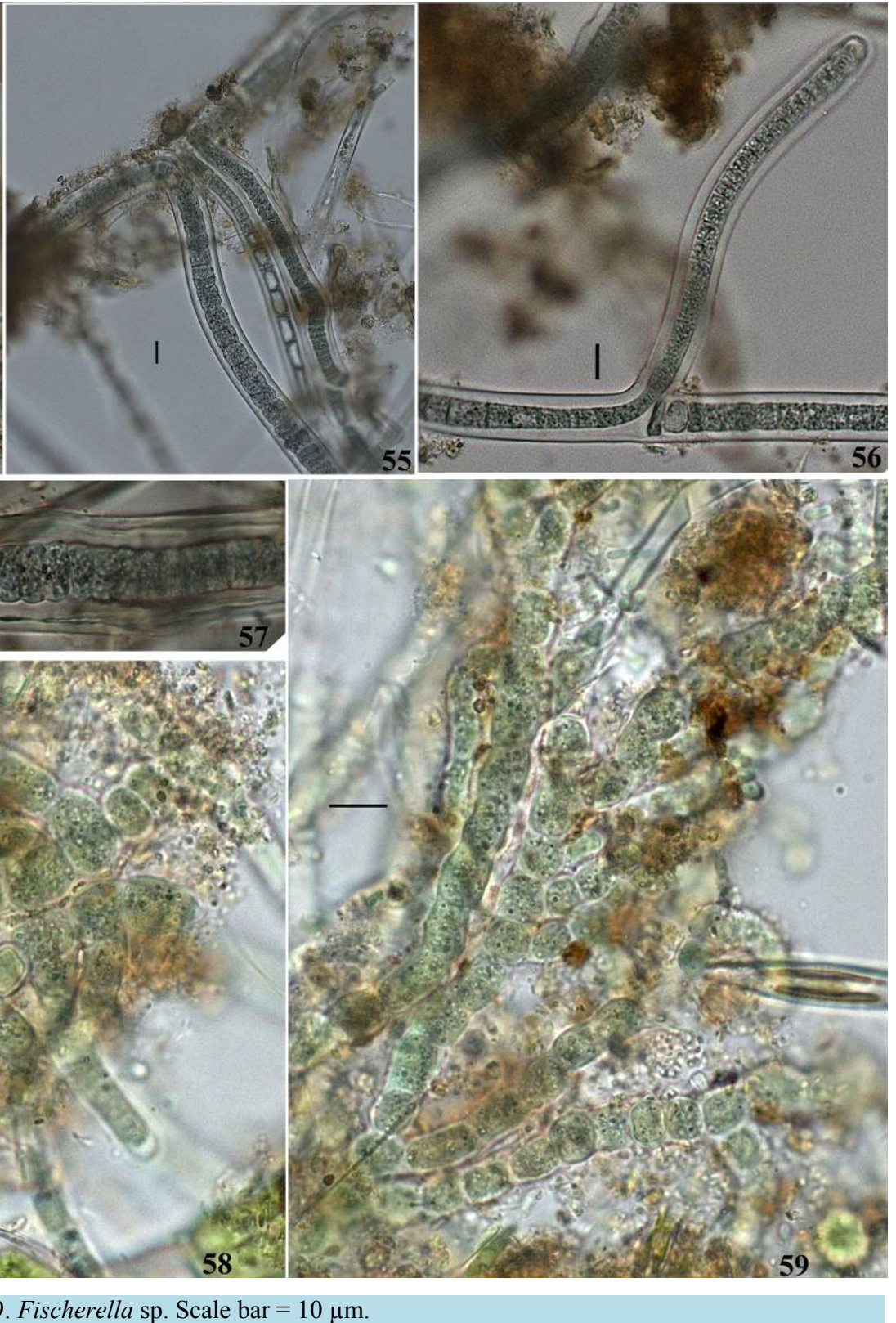

Figure 5. 54-57. Scytonema sp.; 58-59. Fischerella sp. Scale bar $=10 \mu \mathrm{m}$. 


\section{Acknowledgements}

The authors wish to express their gratitude to the Professor Leandro Gonçalves de Oliveira and Ina de Souza Nogueira by cede equipment and physical space of the laboratory analysis and environmental management of water resources (LAMARH), to the Conselho Nacional de Desenvolvimento Cientifico e Tecnológico (CNPq) for the concession of Scientific Initiation scholarship to the second author, and to the company of Goiás Sanitation S/A (Saneago) by cede abiotic dada of Samambaia Reservoir and School of Agronomy and Food Engineering of UFG.

\section{References}

[1] Nascimento, M.A.L.S. (1992) Geomorfologia do Estado de Goiás. Boletim Goiano de Geografia, 12, 1-22.

[2] Felisberto, S.A., Rodrigues, L. and Leandrini, J.A. (2001) Chlorococcales registradas na comunidade perifítica, no reservatório de Corumbá, Estado de Goiás, Brasil, antes e após o represamento das águas. Acta Scientiarum, 23, 275282.

[3] Felisberto, S.A. and Rodrigues, L. (2002) Desmidiales (exceto o gênero Cosmarium) perifíticas no reservatório de Corumbá, Goiás, Brasil. Iheringia, 57, 75-97.

[4] Felisberto, S.A. and Rodrigues, L. (2004) Periphytic desmids in Corumbá Reservoir, Goiás, Brazil: Genus Cosmarium Corda. Brazilian Journal of Biology, 64, 141-150. http://dx.doi.org/10.1590/S1519-69842004000100016

[5] Dunck, B., Nogueira, I.S. and Felisberto, S.A. (2013) Distribution of Epiphytic Algae in Wetlands (Palm Swamps, Cerrado), Brazil. Brazilian Journal of Biology, 73, 331-346.

[6] Dunck, B., Nogueira, I.S. and S.A. (2013) Felisberto. Composição e diversidade de algas perifíticas em veredas sob diferentes impactos antrópicos (Goiás, Brasil). Iheringia Serie Botanica, 68, 237-248.

[7] Leandrini, J.A., Felisberto, S.A. and Dunck, B. (2013) Estrutura da comunidade de algas perifíticas em distintas Veredas no Cerrado goiano. Revista de Biologia Neotropical, 10, 24-35. http://dx.doi.org/10.5216/rbn.v1i1.22313

[8] Souza, D.B.S. and Felisberto, S.A. (2014) Comasiella, Desmodesmus, Pectinodesmus e Scenedesmus na comunidade perifítica em ecossistema lêntico tropical, Brasil Central. Hoehnea, 41, 109-120.

[9] Campo, E., Lezcano, M.A., Agha, R. and Cirés, S. (2013) First TaqMan Assay to Identify and Quantify the Cylindrospermopsin-Producing Cyanobacterium Aphanizomenon ovalisporum in Water. Advances in Microbiology, 3, 430-437. http://dx.doi.org/10.4236/aim.2013.35058

[10] Komárek, J., Sant'Anna, C.L., Bohunicka, M., Mares, J., Hentschke, G.S., Rigonato, J. and Fiore, M.F. (2013) Phenotype diversity and Phylogeny of Selected Scytonema-Species (Cyanoprokaryota) from SE Brazil. Fottea, 13, 173-200.

[11] Komárek, J. and Anagnostidis, K. (1986) Modern Approach to the Classification System of Cyanophytes 2. Chroococcales. Archiv für Hydrobiologie, 73, 157-226.

[12] Komárek, J. and Anagnostidis, K. (1989) Modern Approach to the Classification System of Cyanophytes 4. Nostocales. Archiv für Hydrobiologie, 82, 247-345.

[13] Komárek, J. (2003) Coccoid and Colonial Cyanobacteria. In: Sheath, R.G. and Wehr, J.D., Eds., Freshwater Algae of North America, Elsevier, Amsterdam, 59-116. http://dx.doi.org/10.1016/B978-012741550-5/50004-0

[14] Komárek, J., Komárková, J. and Kling, H. (2003) Filamentous Cyanobacteria. In: Sheath, R.G. and Wehr, J.D., Eds., Freshwater Algae of North America, Elsevier, Amsterdam, 117-196.

[15] Chorus, I. and Bartram, J. (1999) Toxic Cyanobacteria in Water-A Guide to Their Public Health Consequences, Monitoring and Management. Routledge, London and New York.

[16] Brandão, D. and Kravchenko, A. (1997) A biota do Campus Samambaia: História, situação atual e perspectivas. Editora da UFG, Goiânia.

[17] Peel, M.C., Finlayson, B.L. and Mcmahon, T.A. (2007) Updated World Map of the Köppen-Geiger Climate Classification. Hydrology and Earth System Sciences, 11, 1633-1644. http://dx.doi.org/10.5194/hess-11-1633-2007

[18] Azevedo, M.T.P., Sant'Anna, C.L., Senna, P.A.C., Komárek, J. and Komárkova, J. (2003) Contribution to the Microflora of Chroococcalean Cyaniprakaryotes from São Paulo State, Southeast Brazil. Hoehnea, 30, 285-295.

[19] Sant'anna, C.L., Azevedo, M.T.P., Senna, P.A.C., Komárek, J. and Komárková, J. (2004) Planktic Cyanobacteria from São Paulo State, Brazil: Chroococcales. Brazilian Journal of Botany, 27, 213-227. http://dx.doi.org/10.1590/S0100-84042004000200002

[20] Komárek, J. and Anagnostidis, K. (2005) Cyanoprokaryota. Oscillatoriales. Elsevier, München. 
Scientific Research Publishing (SCIRP) is one of the largest Open Access journal publishers. It is currently publishing more than 200 open access, online, peer-reviewed journals covering a wide range of academic disciplines. SCIRP serves the worldwide academic communities and contributes to the progress and application of science with its publication.

Other selected journals from SCIRP are listed as below. Submit your manuscript to us via either submit@scirp.org or Online Submission Portal.
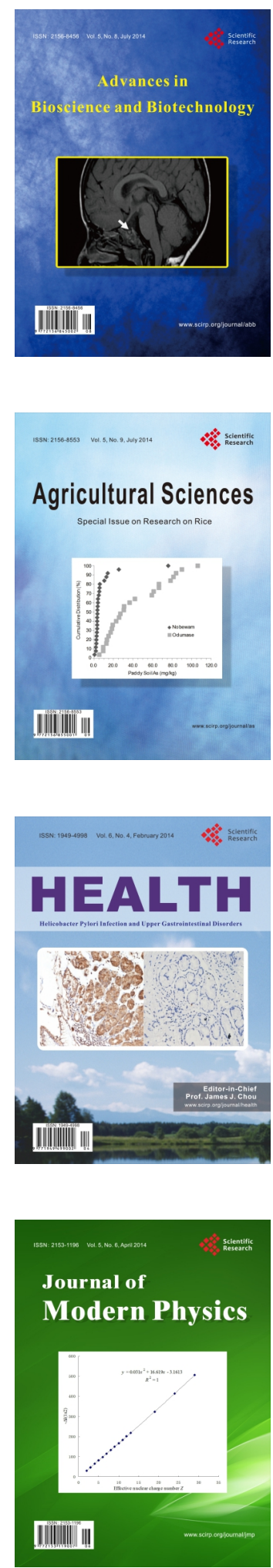
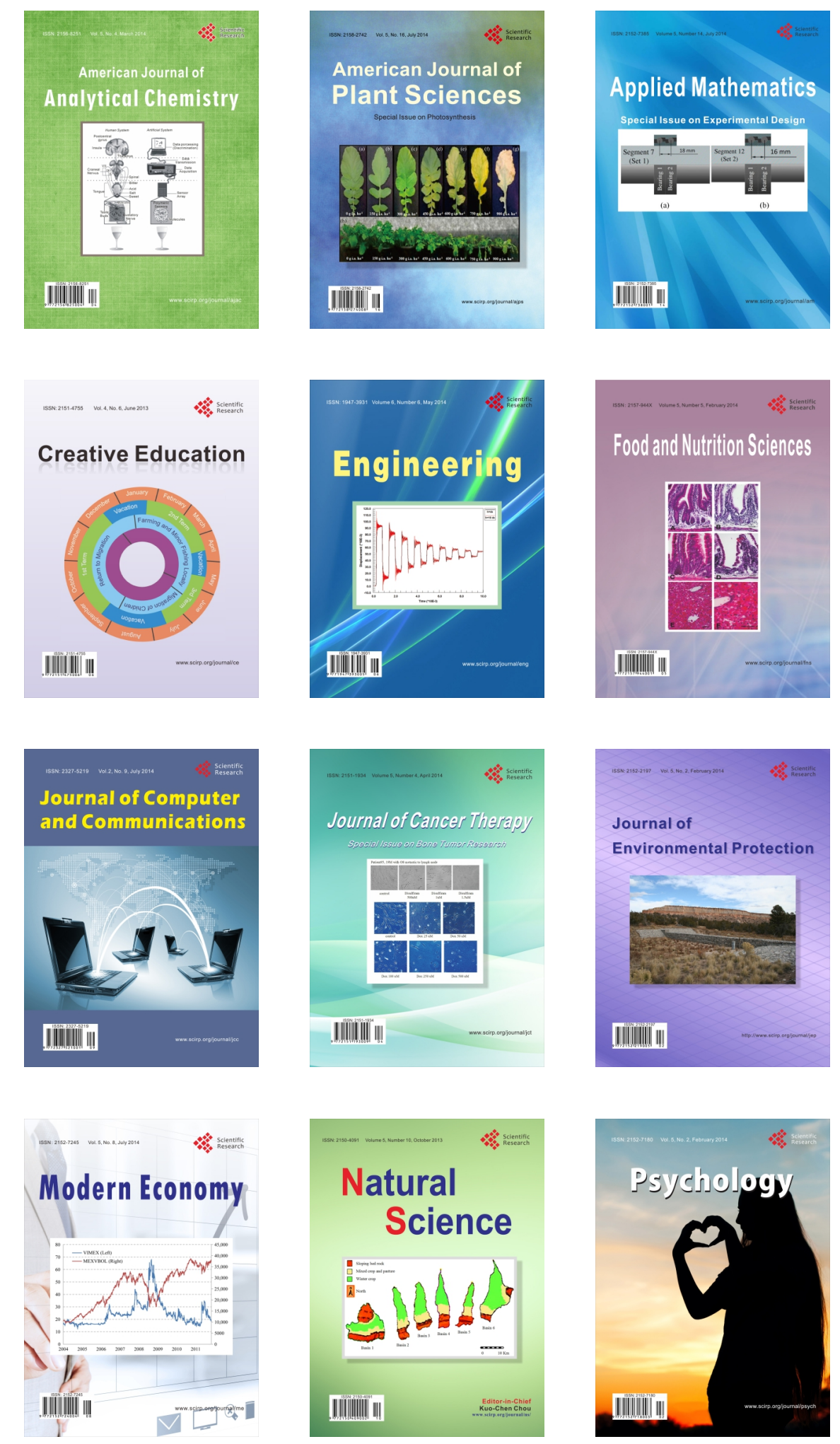